\title{
Electrophysiologically Identified Nigral Dopaminergic Neurons Intracellularly Labeled with HRP: Light-Microscopic Analysis
}

\author{
James M. Tepper, Steven F. Sawyer, and Philip M. Groves \\ Department of Psychiatry, University of California, San Diego, La Jolla, California 92093
}

\begin{abstract}
Intracellular recordings were obtained in vivo from neurons of the rat substantia nigra, pars compacta. Neurons that were identified as dopaminergic by a variety of electrophysiological criteria, including antidromic activation from ipsilateral neostriatum or globus pallidus, were microiontophoretically injected with horseradish peroxidase and examined at the level of the light microscope.
\end{abstract}

Dopaminergic neurons were of medium size and had ovoid, polygonal, or fusiform cell bodies that emitted from 3-6 primary dendrites. Much of the sparse and relatively unbranched dendritic arborization of these neurons remained within pars compacta, except for 1 or 2 large dendrites that were directed ventrally or ventrolaterally into pars reticulata, roughly perpendicular to the plane of the pars compacta. In coronal sections, the dendrites of ovoid- or polygonal-shaped pars compacta neurons were oriented mainly along the dorsoventral axis, whereas fusiform-shaped neurons had dendrites that were oriented primarily mediolaterally.

Although some of the dendrites of dopaminergic neurons exhibited variations in diameter, most were not markedly varicose. Dendrites were sometimes sparsely invested with spinelike appendages or other dendritic extrusions, particularly along their distal portions.

The axons of dopaminergic pars compacta neurons were emitted from primary or proximal secondary dendrites, and were extremely fine processes, $0.5 \mu \mathrm{m}$ or less in diameter. No local axon collaterals were observed.

The dopaminergic nigro-neostriatal projection has been the focus of numerous studies since its discovery over 20 years ago. The great interest in this pathway stems, in large part, from repeated suggestions that abnormal functioning of dopaminergic projections is involved in the symptomatology of several neurological and psychiatric disorders, including Parkinson's disease, tardive dyskinesia, and schizophrenia. Although Parkinsonism has been clearly linked to a loss of dopaminergic input to the neostriatum as a consequence of the degeneration of dopaminergic neurons in the substantia nigra, pars compacta,

\footnotetext{
Received Sept. 15, 1986; revised Apr. 7, 1987; accepted Apr. 7, 1987.

We are grateful to Dr. C. J. Wilson for valuable suggestions during many phases of these experiments, and for his critical appraisal of the manuscript. We thank Drs. M. R. Park and S. J. Young for many helpful comments and Ms. E. Wilson for excellent technical assistance. We are indebted to Mr. Greg Kleinberg and McBain Instruments for the extended loan of the PM10-AK camera system. This research was supported by the Office of Naval Research, Contract N00014-85-k0699, the National Institute on Drug Abuse Grant DA-02854, and Research Scientist Award DA-00079 (to P.M.G.).

Correspondence should be addressed to James M. Tepper, Ph.D., Department of Psychiatry M-003, University of California San Diego, La Jolla, CA 92093.

Copyright (C) 1987 Society for Neuroscience $0270-6474 / 87 / 092794-13 \$ 02.00 / 0$
}

other dopaminergic pathophysiologies are not so clearly due to loss of the dopamine input, and may reflect functional rather than gross structural changes in dopaminergic pathways.

The dependence of dopaminergic neurons on afferent input for "normal" functioning is indicated, in part, by the differences in spontaneous activity observed between in vivo and in vitro preparations. In in vivo extracellular recordings, most nigral dopaminergic neurons fire spontaneously at a slow rate, $0.5-8$ $\mathrm{Hz}$, in an irregular pattern that is frequently punctuated by short bursts of deactivating action potentials, in which the interspike intervals are reduced to as little as 60-70 $\mathrm{msec}$ (Bunney et al., 1973; Wilson et al., 1977b; Tepper et al., 1984; Grace, 1987). Identical parameters of spontaneous activity are also observed with in vivo intracellular recordings (Grace and Bunney, 1980, 1983; Tepper et al., 1986), and the input resistance of these neurons has been shown to be about $31 \mathrm{M} \Omega$ (Gracc and Bunncy, 1983). In contrast, intracellular recordings of pars compacta neurons obtained from in vitro brain slice preparations reveal that these neurons are more hyperpolarized, exhibit significantly less spontaneous activity, possess considerably greater input resistances (Llinás et al., 1984; Kita et al., 1986; Grace, 1987), and lack the characteristic bursting pattern of firing when compared with in vivo recordings (Grace, 1987).

These differences suggest that afferent input may play an important role in the modulation of dopaminergic neuron activity. Moreover, alterations in afferent input could be involved in the etiology of some of the dopaminergic disturbances seen in schizophrenia and the dyskinesias. Although the substantia nigra has been studied in Golgi preparations (Rinvik and Grofová, 1970; Schwyn and Fox, 1974; Juraska et al., 1977), this technique does not allow identification of the target regions of the stained neurons, or correlation of neuronal morphology with electrophysiological properties that identify nigral dopaminergic neurons. Furthermore, the multitude of neurons stained in each section with the Golgi technique usually precludes the complete reconstruction of single neurons followed through serial sections, which results in a rather limited view of the dendritic extent of the neuron. Similar problems arise with immunocytochemical staining of dopaminergic neurons. The technique of identifying the dopaminergic nature and sites of projection of nigral neurons by electrophysiological means, coupled with intracellular injection of HRP, can overcome these drawbacks and provide a more accurate picture of the morphology and dendritic organization of identified nigral dopaminergic neurons. As a first step towards identifying and characterizing the functional afferents to nigral dopaminergic neurons, the present study examines, at the light-microscopic level, the morphology of electrophysiologically identified nigrostriatal neurons from rat pars compacta intracellularly labeled with HRP. Subsequent reports 
will describe the neurophysiological responses to afferent stimulation, and the ultrastructure and synaptic contacts of these nigral neurons. A preliminary report of these data has previously appeared (Tepper et al., 1986).

\section{Materials and Methods}

Animal preparation. The subjects for this study were male SpragueDawley rats, weighing between 225 and $325 \mathrm{gm}$ at the time of recording. Rats were anesthetized with urethane $(1.3 \mathrm{gm} / \mathrm{kg}$, i.p.) and fixed in a stereotaxic apparatus according to the atlas of König and Klippel (1963). All wound margins and points of contact between the rat and the stereotaxic apparatus were thoroughly infiltrated with lidocaine ointment $(5 \%)$ or solution $(2 \%)$. The scalp was removed and small burr holes drilled for the insertion of bipolar stimulating electrodes into the ipsilateral anterior-lateral neostriatum (A: 1.0 from bregma, L: 3.7, V: 4.0 from surface), and ipsilateral globus pallidus (A: $-1.3, \mathrm{~L}: 3.2, \mathrm{~V}: 6.0$ ). Stimulating electrodes were affixed to the skull with cyanoacrylate glue and dental cement. A larger hole was drilled over the substantia nigra, centered at A: 2.1 from lambda and L: 2.3. To reduce respiratory and cardiovascular pulsation, animals were suspended via $\mathrm{C} 2$ vertebral and tail clamps, and the atlanto-occipital membrane was punctured and some cerebrospinal fluid allowed to drain. Body temperature was maintained at $37 \pm 1^{\circ} \mathrm{C}$, and the electrocardiogram was constantly monitored on an auxiliary oscilloscope. A well of dental cement was constructed around the recording site and was filled with a mixture of paraffin and paraffin oil after the recording electrode was inserted, in order to prevent drying and further increase stability.

Electrodes and stimulation. Micropipettes for intracellular recording and HRP injection were fashioned from $2.0 \mathrm{~mm}$ OD WPI Kwik-Fill capillary glass on a Narishige vertical pipette puller, and broken back under microscopic control to achieve final tip diameters of $0.2-0.6 \mu \mathrm{m}$. Electrodes were filled with a solution of 5\% HRP (Sigma type VI) in $0.5 \mathrm{M}$ potassium methyl sulfate buffered with $0.05 \mathrm{M}$ Tris $(\mathrm{pH} 7.6)$, and possessed in vivo impedances ranging from 35 to $80 \mathrm{M} \Omega$. Electrode filling was facilitated by the insertion of a tungsten wire "twirler," etched to a tip diameter of less than $1 \mu \mathrm{m}$.

Extracellular electrical stimulation was delivered through bipolar enamel-coated stainless steel electrodes with tip separations of less than $250 \mu \mathrm{m}$ (Tepper ct al., 1984). Stimuli were generated by a Grass S-88 or SD-9 stimulator and consisted of monophasic square-wave pulses, $20-300 \mu \mathrm{sec}$ in duration and $0.3-2.0 \mathrm{~mA}$ in amplitude. Signals from the recording electrode were amplified by a WPI M-707A preamplifier, digitized on-line by a Nicolet 2090 digital oscilloscope, fed to a PDP11/03 microcomputer, and stored on a floppy disk for off-line analysis.

Identification and intracellular labeling of dopaminergic neurons. Neurons in the substantia nigra, pars compacta were identified as dopaminergic on the basis of their extracellularly and intracellularly recorded waveform, firing rate and pattern, and the characteristics of their antidromic responses to stimulation of the ipsilateral neostriatum and/ or globus pallidus (Bunney et al., 1973; Wilson et al., 1977b; Deniau et al., 1978; Guyenet and Aghajanian, 1978; Grace and Bunney, 1983; Tepper et al., 1984, 1986). Once stable intracellular recording was achieved (resting membranc potential, $40 \mathrm{mV}$ or greater, with spontaneous or evoked spikes of $45 \mathrm{mV}$ or greater), attempts were made to activate the neuron antidromically from neostriatum or pallidum. Responses were identified as antidromic based on constant latency and collision with spontaneous or intracellularly evoked spikes (Tepper et al., 1984). If stable recording conditions persisted throughout the electrophysiological characterization, HRP was injected iontophoretically through the bridge circuitry of the WPI preamplifier, with a 50\% duty cycle of $300 \mathrm{msec}$ depolarizing pulses at 2-6 nA for 3-15 min. Neuronal activity was monitored throughout the procedure, and HRP injection was terminated immediately upon signs of deteriorating recording conditions to prevent artifactual staining. Only dopaminergic neurons that responded antidromically to stimulation of neostriatum or globus pallidus were injected with HRP.

Tissue preparation. Postinjection survival times ranged from 30 to $150 \mathrm{~min}$, after which animals were administered a lethal dose of urethane and perfused transcardially with $50-100 \mathrm{ml}$ of oxygenated rat Ringer's ( $\mathrm{pH} 7.2$ ), followed by $300 \mathrm{ml}$ of $1 \%$ paraformaldehyde $-2 \%$ gluteraldehyde buffered with $0.15 \mathrm{~m}$ phosphate, $\mathrm{pH} 7.2$. Brains were removed and stored for up to $6 \mathrm{~d}$ in cold $0.15 \mathrm{M}$ phosphate buffer, and $60 \mu \mathrm{m}$ coronal sections through the substantia nigra were cut in cold phosphate buffer on a Vibratome. Sections were reacted for the presence
A

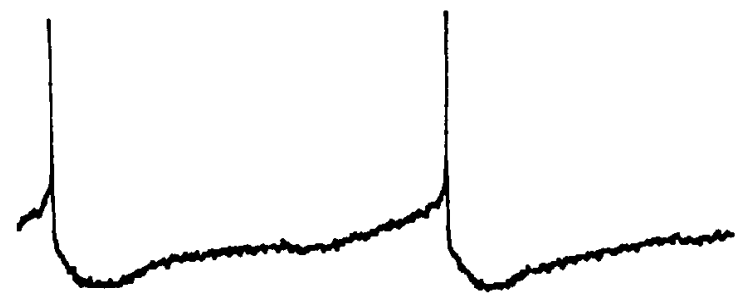

B

C
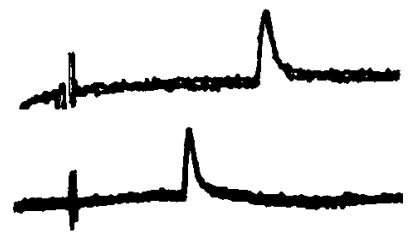

D

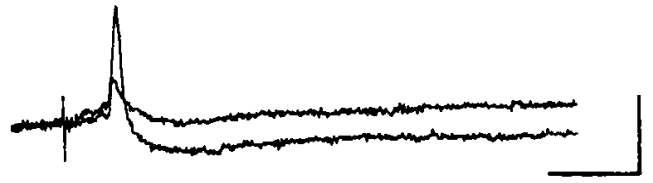

Figure 1. Elcctrophysiological identification of dopaminergic neurons in substantia nigra pars compacta. $A$, Spontaneous activity. Note the slow ramp depolarization preceding each spike and the large, longlasting afterhyperpolarization. $B$, Antidromic activation from ipsilateral neostriatum. Superimposition of 5 sweeps. All responses consist of the IS spike only. $C$, Antidromic activation from ipsilateral globus pallidus. Superimposition of 5 sweeps. All responses consist of the IS spike only. $D$, Antidromic activation from ipsilateral neostriatum illustrating absence of spike afterhyperpolarization following IS spike, though it occurs following a full spike. Superimposition of 2 sweeps. Calibration in $B$ applies to $A-C$. Vertical: $30 \mathrm{mV}$ throughout. Horizontal: $100 \mathrm{msec}(A)$ $5 \mathrm{msec}(B, C), 10 \mathrm{msec}(D)$.

of HRP by the glucose oxidase method of Itoh et al. (1979) using 3'-3diaminobenzidine as a chromagen, postfixed in $0.5 \%$ osmium tetroxide for $60 \mathrm{~min}$, dehydrated in an ascending series of alcohols and acetone, stained with uranyl acetate, and embedded in Spurr's medium between Teflon-coated glass slides and coverslips for sequential light and electron microscopy, as described by Wilson and Groves (1979).

Sixty or $100 \mu \mathrm{m}$ frozen sections through the ipsilateral ncostriatum and globus pallidus were stained with neutral red in order to verify the location of the stimulating electrodes.

Light-microscopic examination. Sections containing the substantia nigra were examined on a Leitz Ortholux II microscope, and HRP-filled neurons and their processes were drawn with the aid of a Leitz drawing tube using a $50 \times$ oil-immersion objective. Somatic sizes were measured along the long axis of each neuron, and perpendicular to the long axis. Camera lucida drawings of HRP-filled neurons were reconstructed from 4-9 serial sections. Areas of interest were photographed with an Olympus PM10-AK automatic camera system.

\section{Results}

\section{Electrophysiological identification of nigral dopaminergic} neurons

Intracellular recordings were obtained from 25 neurons that fulfilled previously established electrophysiological criteria for identification as nigral dopaminergic projection neurons (Bunney et al., 1973; Deniau et al., 1978; Guyenet and Aghajanian, 1978; Grace and Bunney, 1983; Grace, 1987). Ten of these neurons were injected with HRP, and 8 were successfully recovered in suitable condition for further light-microscopic analysis. In addition, 3 neurons with markedly different electrophysiological properties, consistent with their identification as nondopaminergic nigral, pars reticulata neurons (Deniau et al., 1978; Guyenet and Aghajanian, 1978; Grofová et al., 1982) were injected with HRP and successfully recovered. The fol- 


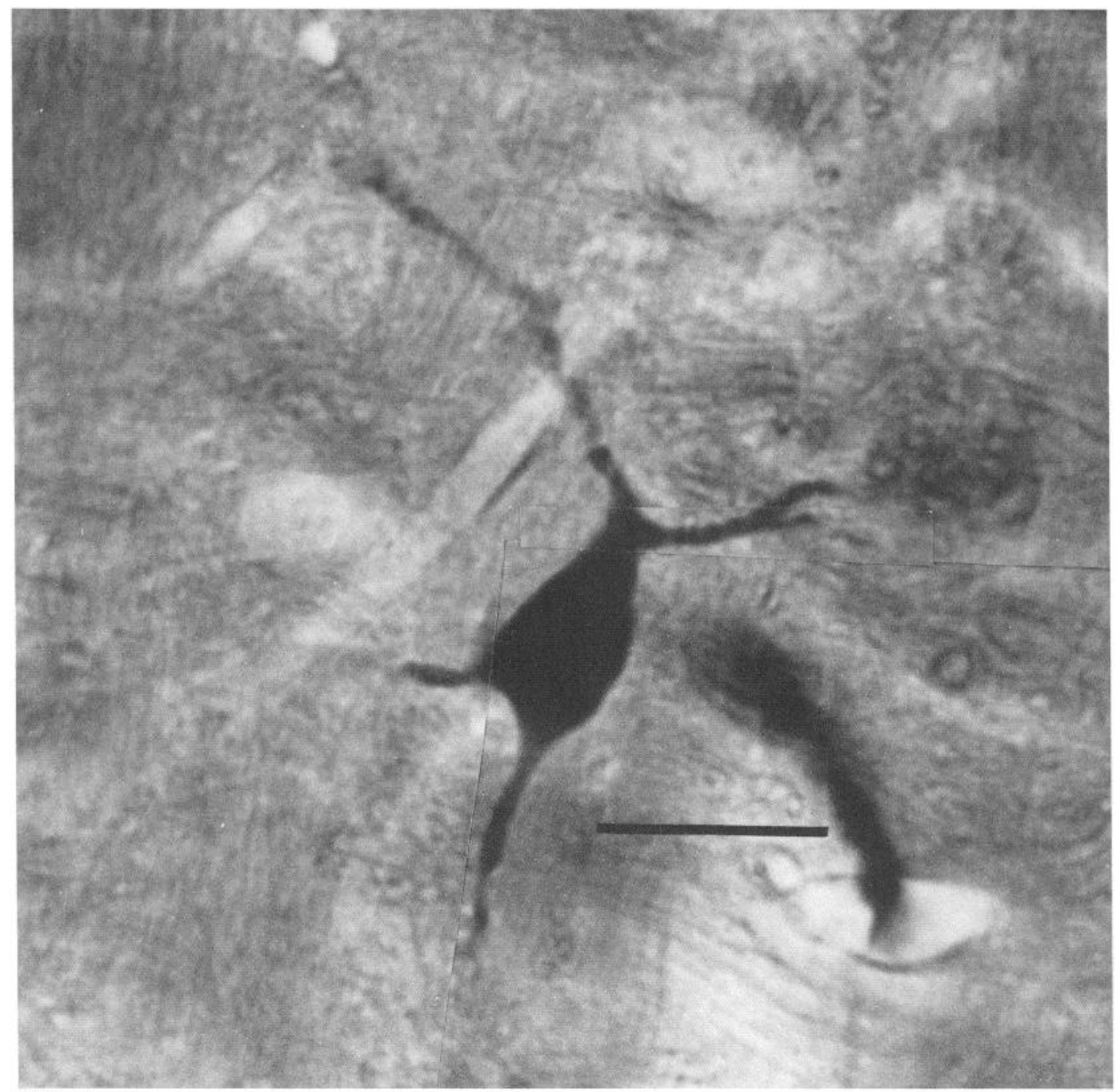

Figure 2. Composite photomicrograph of the soma and proximal dendrites of a typical HRP-filled electrophysiologically identified rat pars compacta dopaminergic nigrostriatal neuron. Top is dorsal, left is lateral. Scale, $25 \mu \mathrm{m}$.

lowing electrophysiological description is based solely on the 8 recovered dopaminergic neurons, but is representative of all 25 neurons recorded. Seven of the 8 neurons were spontaneously active and fired at slow rates $(<6$ spikes/sec) with characteristic long-duration action potentials $(>2.5 \mathrm{msec})$, arising from slow ramp depolarizations, as previously reported for identified nigral dopaminergic neurons (Grace and Bunney, 1983). Spontaneous spikes were followed by a prominent, long-lasting afterhyperpolarization, also typical of nigral dopaminergic neurons, as shown in Figure 1A. The identity of all HRP-filled dopaminergic neurons in this report was further confirmed by antidromic activation from ipsilateral neostriatum and/or globus pallidus. The antidromic responses elicited by neostriatal stimulation were of long latency $(13.9 \pm 0.8 \mathrm{msec}$, mean \pm SEM), and typically consisted of an initial segment (IS) spike only, as shown in Figure $1 B$. Antidromic responses elicited by pallidal stimulation had a shorter latency $(7.1 \pm 0.7 \mathrm{msec}$, and also usually consisted of IS spikes only, as illustrated in Figure $1 C$. Although antidromically evoked full spikes, like spontaneous spikes, were followed by a prolonged spike afterhyperpolarization, this was not observed following IS-only antidromic spikes, as shown in Figure $1 D$. The 3 nondopaminergic pars reticulata neurons exhibited markedly different electrophysiological properties; these neurons fired spontaneously in excess of 15 spikes/ $\mathrm{sec}$, the action potential durations were less than $1.5 \mathrm{msec}$, the prominent long-lasting spike afterhyperpolarization was absent, and these neurons could not be antidromically activated from neostriatum or globus pallidus.

\section{Light-microscopic observations}

The 8 HRP-filled dopaminergic projection neurons were distributed across the rostrocaudal and mediolateral extent of the substantia nigra, pars compacta. The cell bodies were mediumsized, $24.8 \pm 1.7 \times 12.7 \pm 3.4 \mu \mathrm{m}$, and were ovoid, polygonal, or fusiform in shape. The cell body and proximal dendrites of a representative pars compacta nigrostriatal neuron are shown in Figure 2. Pars compacta somata emitted 3-6 thick primary dendrites, $2-6 \mu \mathrm{m}$ in diameter, radiating in all directions, which gave rise to a relatively sparse arborization of thinner secondary and tertiary dendrites. Higher-order dendritic branching was, in general, not observed. Camera lucida drawings of 4 representative pars compacta neurons, along with their relative locations within substantia nigra, are presented in Figures 3-6.

Neurons with ovoid or polygonal cell bodies had dendritic 
arbors whose major extent tended to be oriented dorsoventrally, roughly perpendicular to the surface of pars compacta (Figs. 3, 4). In contrast, fusiform neurons tended to have more elongated lateral dendrites, coursing parallel to the surface of pars compacta, and appeared to lack dorsally directed dendrites (Fig. 5). A significant proportion of the dendritic arborization of the dopaminergic neurons remained within the confines of the pars compacta. In coronally sectioned material, dendrites emerging from the lateral side of the soma traveled in a dorsolateral direction, while those emanating from the medial aspect of the cell ran in a ventromedial direction, thus closely paralleling the borders of the pars compacta. In fusiform neurons, the medial and lateral dendrites were thick and long, giving the neuron a flattened and elongated dendritic field within pars compacta of well over a millimeter in mediolateral extent (cf. Fig. 5). In the most dorsally situated neurons, the longest of the dorsal or dorsolateral dendrites penetrated the most ventral portions of the medial lemniscus.

Pars compacta neurons usually sent 1 or 2 dendrites coursing ventrally or ventrolaterally, roughly perpendicular to the surface of pars compacta, often very deep into pars reticulata. These ventral dendrites were usually the largest emitted by the cell, both in diameter and overall length. In several cases, the ventral dendrites terminated among the fibers of the crus cerebri (cf. Figs. 4, 7A).

Most pars compacta neurons also possessed a number of rather short dendrites, radiating dorsally and laterally around the soma. Even in a complete reconstruction of neurons with dendritic arbors occupying shells of up to $500 \times 1500 \mu \mathrm{m}$, these dorsal and lateral dendrites, some of which were quite thick, usually terminated abruptly, in or just dorsal to pars compacta, within $250 \mu \mathrm{m}$ of the cell body.

Although some of the dendrites of pars compacta neuronsparticularly the thinner, more distal segments-appeared slightly varicose, the vast majority of the dendrites of these neurons were of relatively constant diameter, as shown in Figure $7, A-$ $C$, and in the composite microphotographs in Figures 10 and 11. There appeared to be some variability in the morphology of dendrites arising from single dopaminergic neurons; most of these neurons possessed 1 or 2 dendrites that appeared more varicose than other dendrites of similar diameter from the same neuron. In some cases, dopaminergic dendrites exhibited some variation in diameter, but this usually took the form of irregularly spaced constrictions in dendritic caliber, as shown in Figure $7, D, E$. This was in contrast to the sometimes large and punctate varicose enlargements often observed in the dendrites of the 2 nondopaminergic pars reticulata ncurons injected with HRP, illustrated in Figure $7, F, G$. Nondopaminergic pars reticulata neurons also differed from dopaminergic neurons by virtue of a considerably more extensive dendritic arborization, characterized by thicker and more highly branched higher-order dendrites.

Some dopaminergic dendrites were sparsely invested with spinelike appendages, or other shorter, squal dendritic extrusions. In most cases, the spinelike structures appeared on the distal regions of secondary and tertiary dendrites, both within pars compacta and pars reticulata, as shown in Figure $8, A-C$. In one compacta neuron with an unusually extensive dendritic field (Figs. 6, 10), both spinelike appendages and dendritic blebs were present on 2 primary dendrites as well, as illustrated in Figure 8, $D-F$.

The axons of pars compacta neurons were tentatively iden-

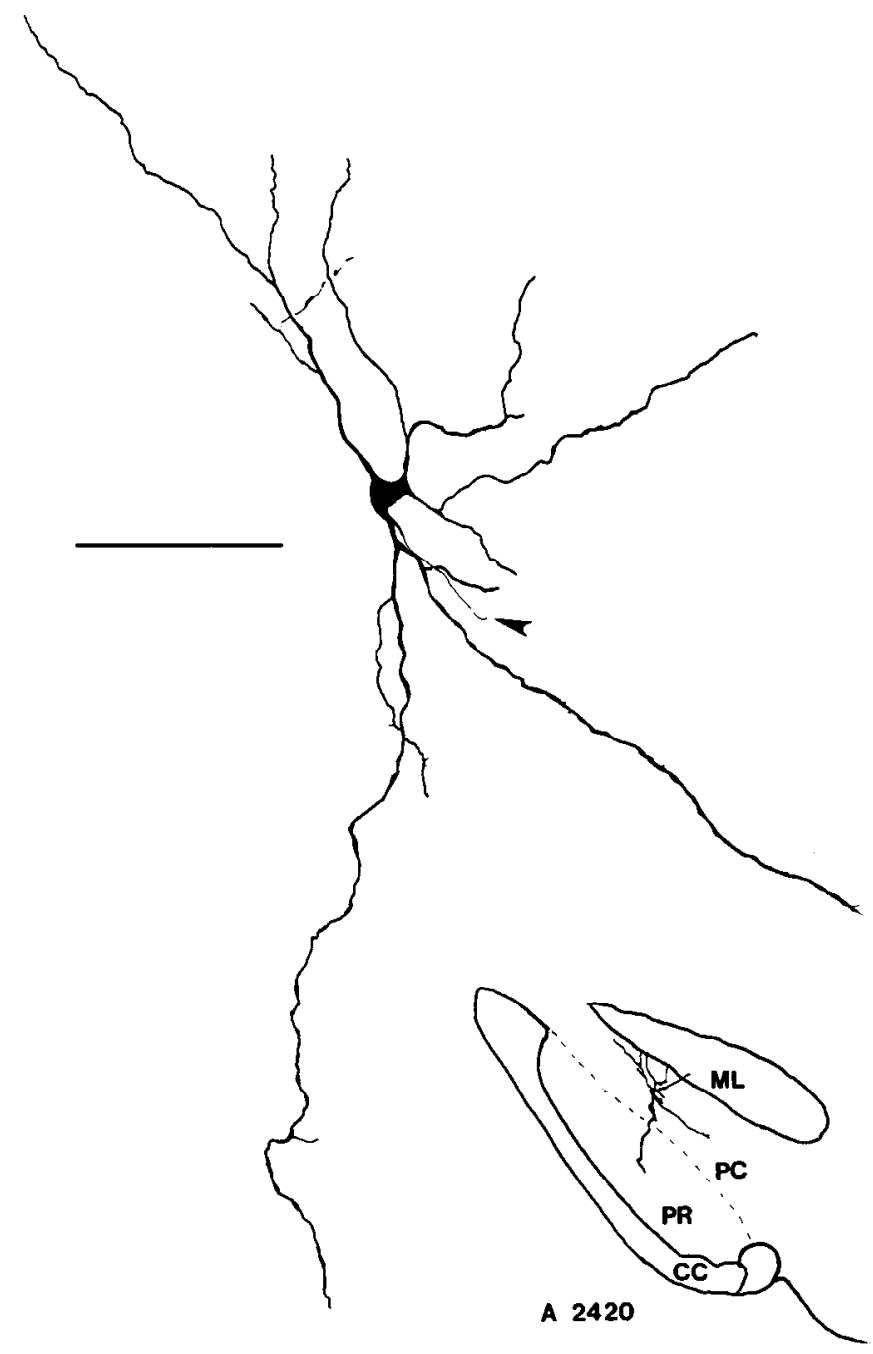

Figure 3. Camera lucida reconstruction of an HRP-filled pars compacta dopaminergic neuron, antidromically activated from neostriatum. A photomontage of this neuron appears in Figure 11. Inset is drawn approximately to scale and illustrates the location of each neuron's dendritic field within substantia nigra. Coordinates refer to location of the cell bodies according to the atlas of König and Klippel (1963). The arrow points to the axon. $M L$, medial lemniscus; $P C$, pars compacta; $P R$, pars reticulata. Scale, $100 \mu \mathrm{m}$.

tified on the basis of their very fine, nontapering diameter, which was considerably smaller than that of any of the higher-order dendrites emitted by the neuron, and by the fact that only one such process was observed emanating from each neuron. The axon usually emerged at an acute angle from a primary or proximal secondary dendrite within approximately $30-80 \mu \mathrm{m}$ from the soma, and was initially directed medially or ventromedially before turning rostrally. The axons were of extremely fine caliber, and appeared to be less than $0.5 \mu \mathrm{m}$ in diameter, as shown in Figure 9. These axons sometimes exhibited irregularly spaced varicosities at distances of over $100 \mu \mathrm{m}$ from their point of origin. No local collaterals were observed, even in axons followed for over $200 \mu \mathrm{m}$.

\section{Discussion}

\section{Identification of neuron type}

Substantia nigra dopaminergic neurons possess a characteristic electrophysiological profile that allows them to be unambiguously identified and distinguished from other, nondopaminergic 
Figure 4. Camera lucida reconstruction of an HRP-filled identified dopaminergic ncuron, antidromically activated from both neostriatum and globus pallidus. Scale, $100 \mu \mathrm{m}$. Inset, see Figure 3.

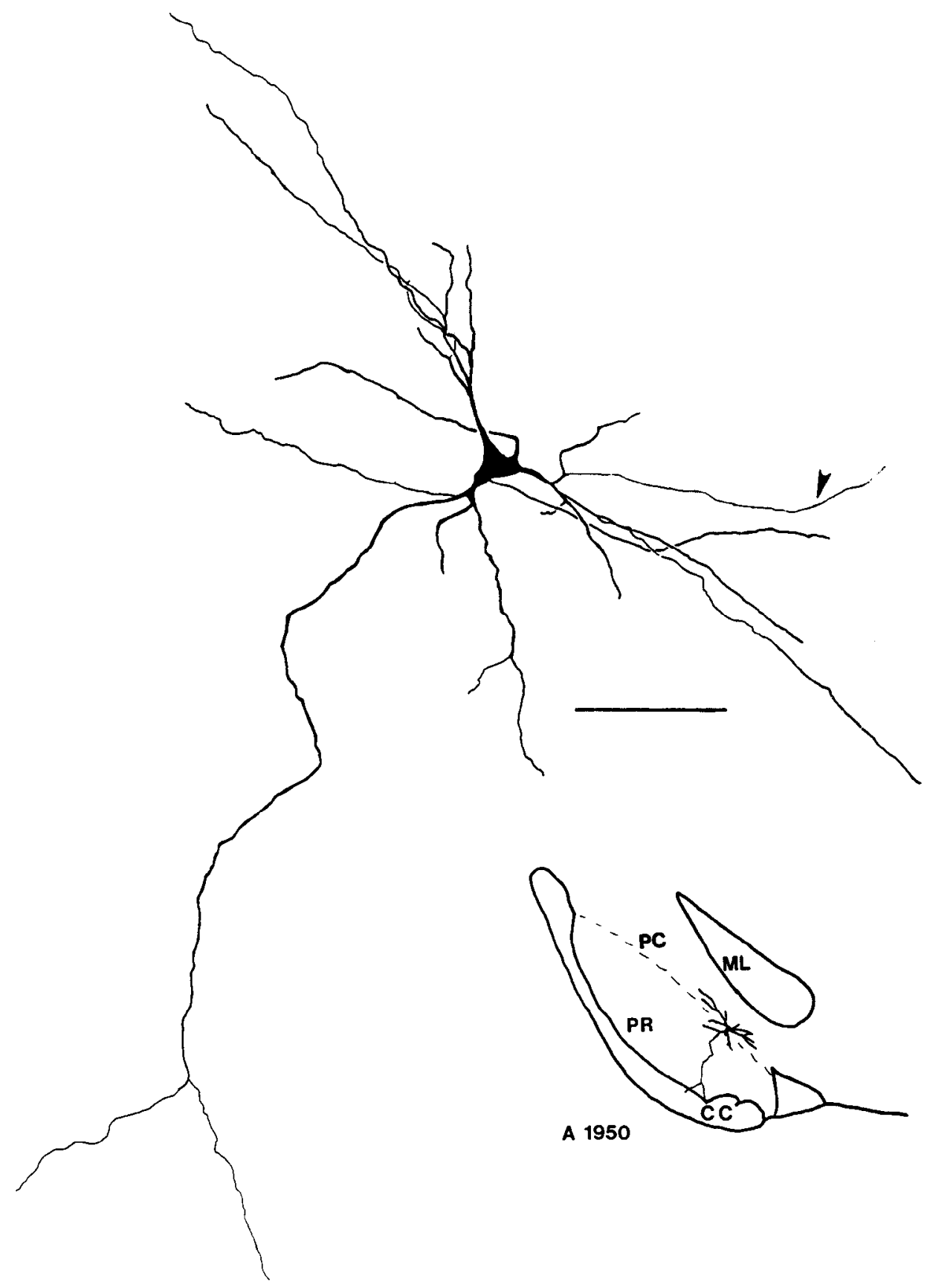

neurons in the substantia nigra and surrounding regions (Bunney et al., 1973; Wilson et al., 1977b; Deniau et al., 1978; Guyenet and Aghajanian, 1978; Grace and Bunney, 1980, 1983; Grofová et al., 1982; Tepper et al., 1984, 1986; Grace, 1987). All pars compacta neurons described in this report were antidromically activated from ipsilateral neostriatum and/or globus pallidus. The antidromic responses elicited from both sites usually consisted of the IS spike only, with full IS-somato-dendritic (SD) antidromic responses occurring only occasionally, even at a stimulation rate of $1 \mathrm{~Hz}$. This feature of the antidromic responses is typical of nigral dopamine neurons recorded with both extracellular (Deniau et al., 1978; Guyenet and Aghajanian, 1978; Tepper et al., 1984) and intracellular (Grace and Bunney, 1983; Tepper et al., 1986) techniques. Although nondopaminergic pars reticulata neurons can occasionally be antidromically activated from neostriatum, these antidromic responses consist of a full spike with no evidence of IS-SD fractionation, except at high rates of stimulation (Deniau et al., 1978; Guyenet and Aghajanian, 1978). The antidromic responses of the nigrostriatal and nigropallidal neurons reported in the present study occurred at long latencies (nigrostriatal: $13.9 \pm 0.8 \mathrm{msec}$; nigropallidal: $7.1 \pm 0.7 \mathrm{msec}$ ), corresponding to estimated straightline conduction velocities in the range of $0.5-0.7 \mathrm{~m} / \mathrm{sec}$. These estimated conduction velocities match those of identified dopaminergic neurons in previous studies (Deniau et al., 1978; Guyenet and Aghajanian, 1978; Grace and Bunney, 1983; Tepper et al., 1984), and are at least 2-3 times slower than those reported for nondopaminergic nigral neurons projecting to striatum, thalamus, or tectum (Deniau et al., 1978; Guyenet and Aghajanian, 1978). 


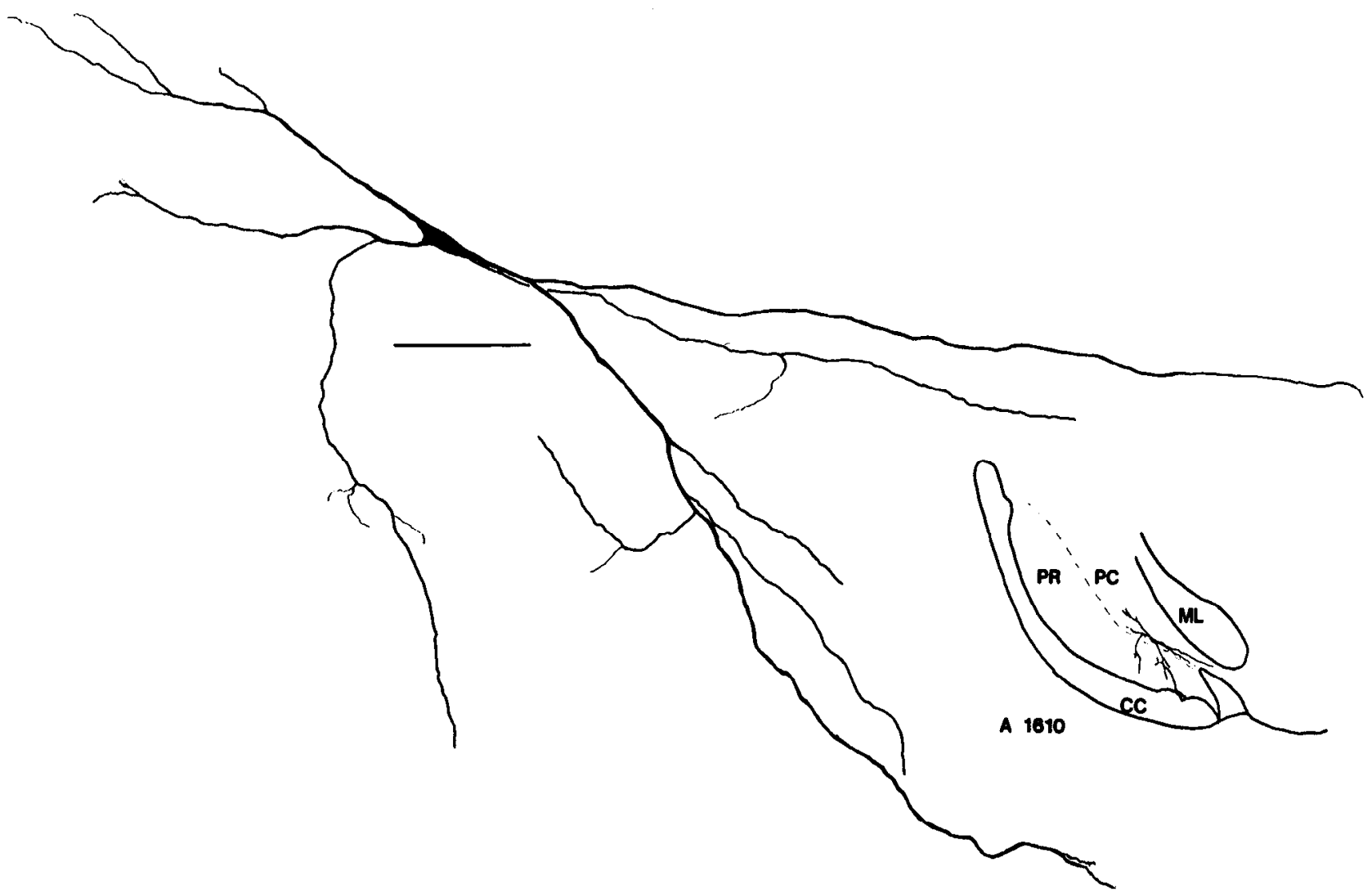

Figure 5. Camera lucida reconstruction of an HRP-filled identified dopaminergic neuron, antidromically activated from neostriatum. Note the predominance of the long, relatively unbranched lateral dendrites. Scale, $100 \mu \mathrm{m}$. Inset, see Figure 3.

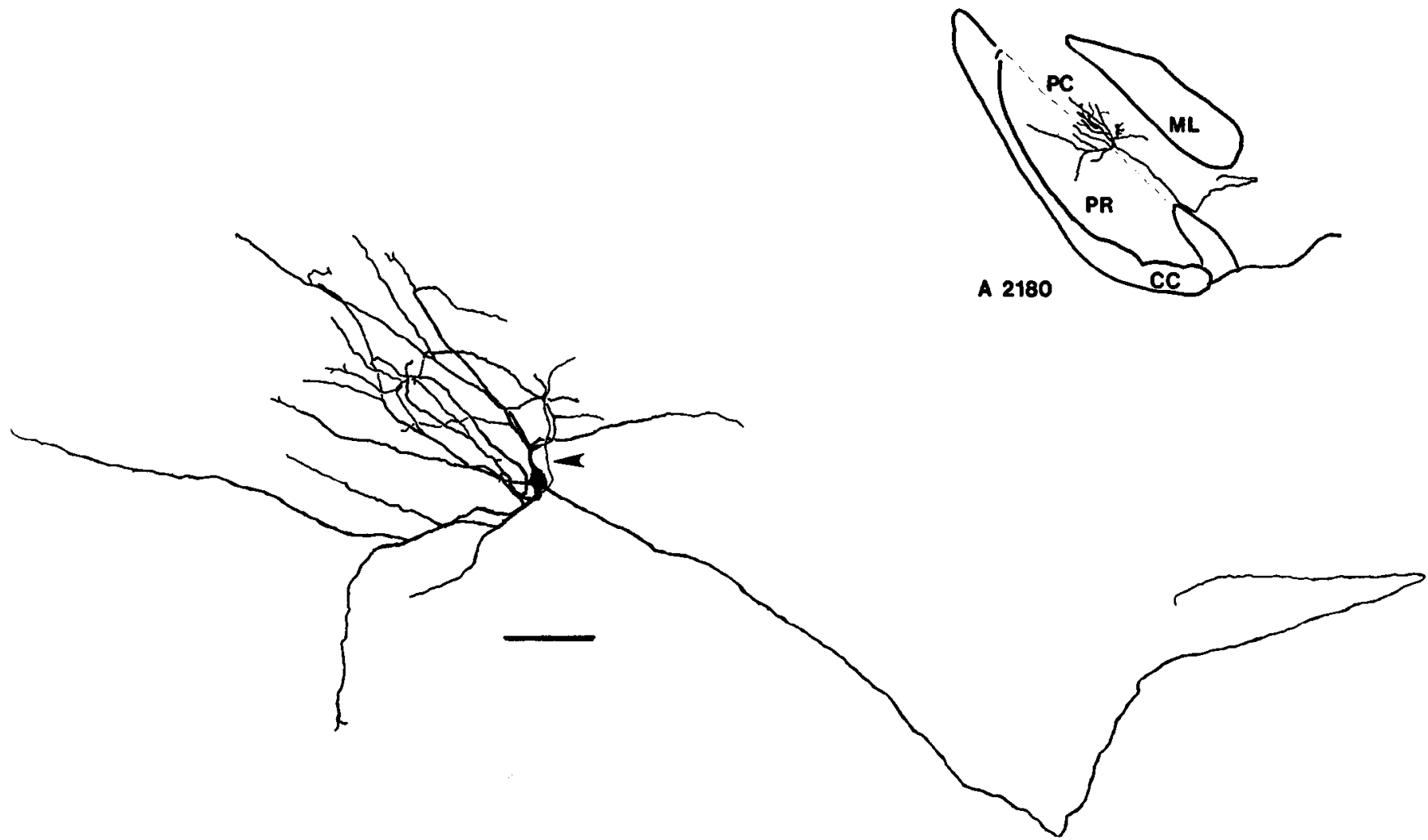

Figure 6. Camera lucida reconstruction of an HRP-filled dopaminergic neuron, antidromically activated from neostriatum. The thick, medially directed dendrite remains within pars compacta, closely following the border between compacta and reticulata until it exits the substantia nigra proper and extends into the ventral tegmental area. It is unbranched throughout its course, which extends over $1500 \mu \mathrm{m}$. Scale, $100 \mu \mathrm{m}$. Inset, see Figure 3. 

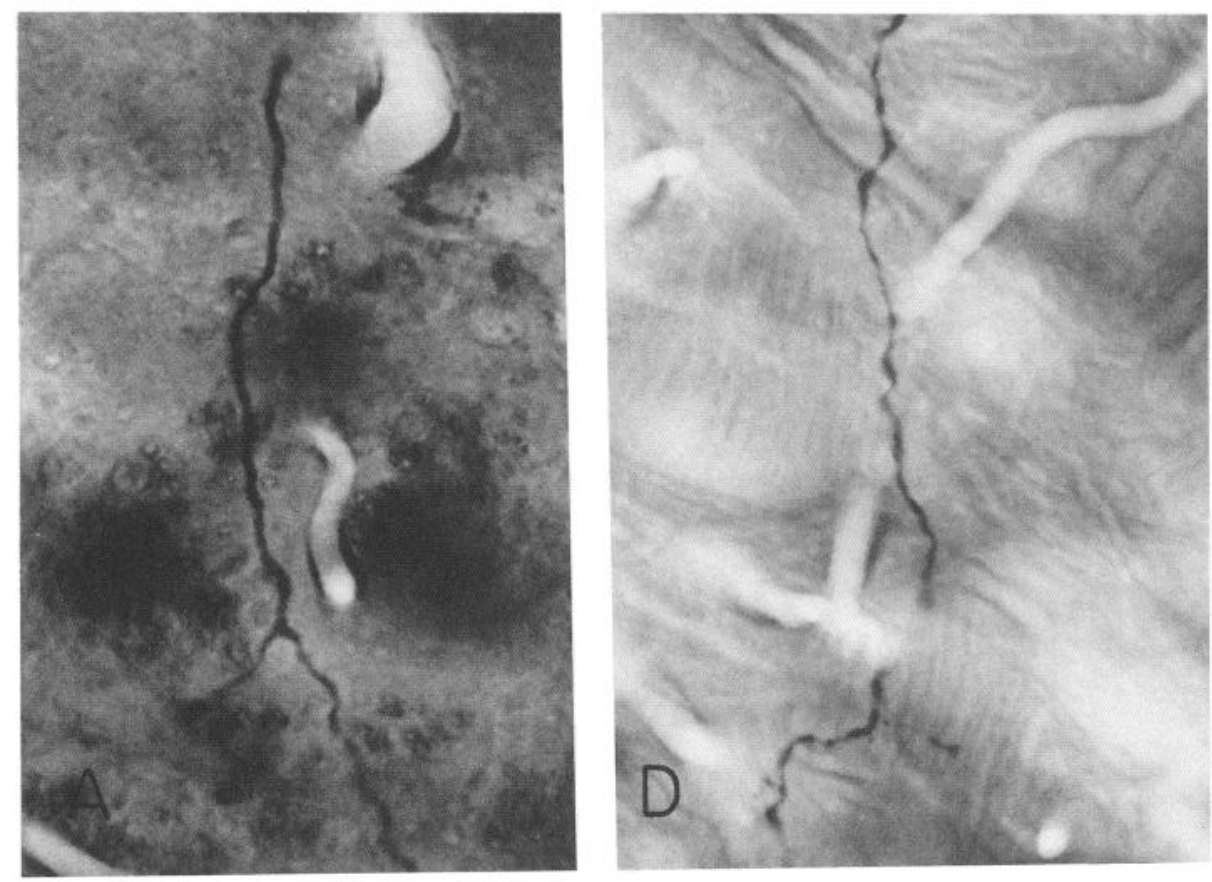

Figure 7. HRP-filled nigral dendrites. $A$, Distal region of a ventral dendrite of a pars compacta dopaminergic neuron terminating among fibers of the crus cerebri at the base of pars reticulata. Note the constant diameter of the dendrite. $B$, Thick secondary dendrite of a fusiform pars compacta dopaminergic neuron coursing medially in pars compacta, exhibiting constant diameter. $C$, Primary lateral dendrite of a dopaminergic neuron in pars compacta. $D$, Distal region of a ventral dendrite of a pars compacta dopaminergic neuron displays variations in diameter, consisting of irregularly spaced constrictions in caliber. $E$, Secondary dorsal dendrite of a dopaminergic neuron in pars compacta exhibiting irregularly spaced constrictions in diameter. $F, G$, Two different secondary dendrites of nondopaminergic pars reticulata neurons exhibiting large varicosities. Compare with dopaminergic dendrites in $D$ and $E$. Scale, $20 \mu \mathrm{m}(A-D, F, G) ; 10$ $\mu \mathrm{m}(E)$.
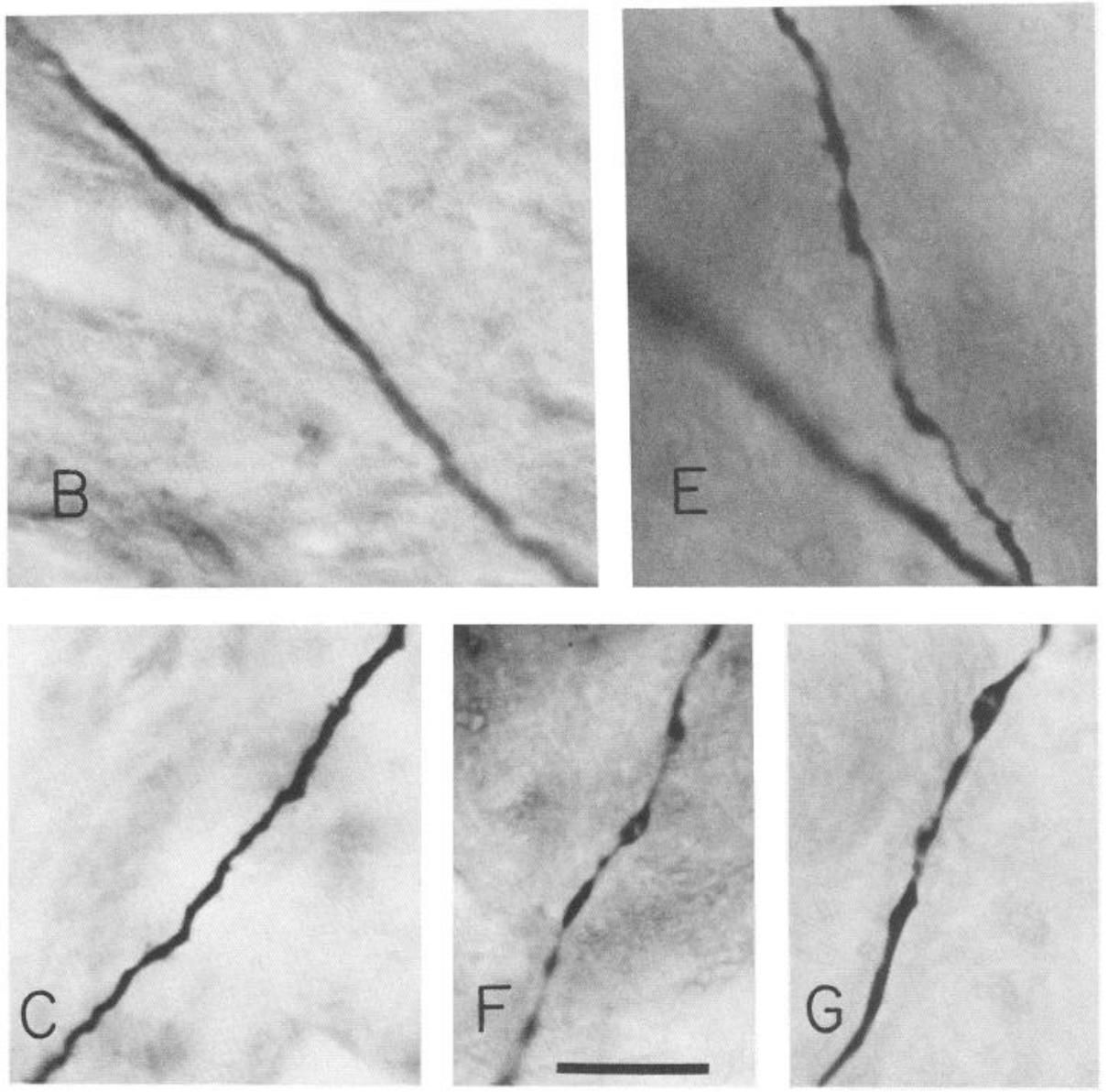

Seven of the 8 pars compacta neurons were spontaneously active, firing in a slow, irregular pattern of approximately 1-6 spikes/sec, with long-duration action potentials exhibiting very large and long-lasting spike afterhyperpolarizations. These properties are also characteristic of nigral neurons identified as do- paminergic by a variety of extracellular and intracellular electrophysiological and histochemical techniques (Guyenet and Aghajanian, 1978; Grace and Bunney, 1980, 1983), in contrast to nondopaminergic pars reticulata neurons, which display significantly higher firing rates, exhibit action potentials of shorter 

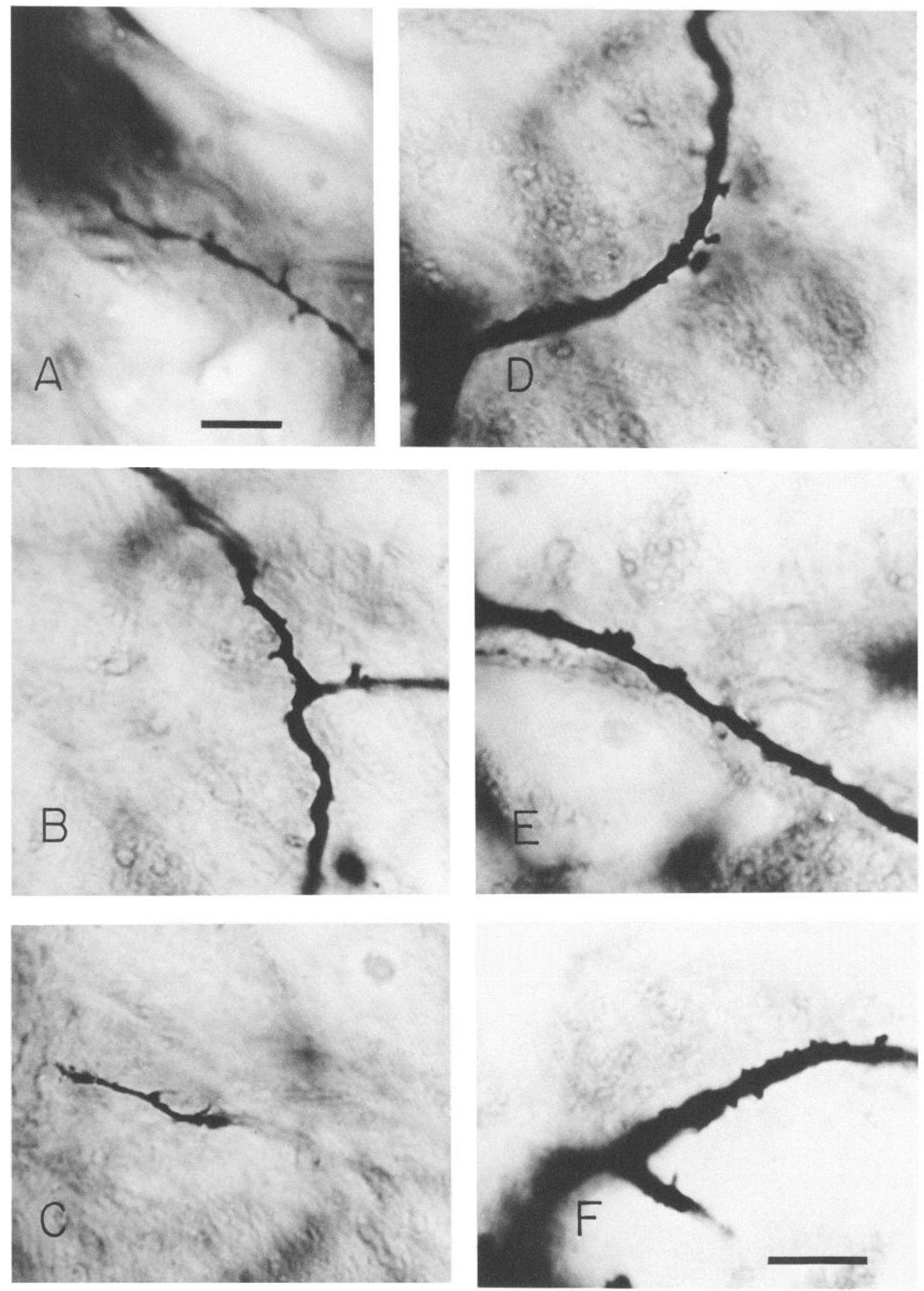

Figure 8. Dendritic specializations of dopaminergic dendrites. $A-D$, Spinelike appendages on secondary dendrites. $E, F$, Squat, bleblike dendritic extrusions on primary dendrites. Scale in $A, 10 \mu \mathrm{m}$. Scale in $E, 10 \mu \mathrm{m}(B-E)$. 
Figure 9. Photomontage illustrating the initial segment and axonal trajectory (white arrows) of a nigrostriatal dopaminergic neuron. Top is dorsal, left is lateral. Scale, $30 \mu \mathrm{m}$.

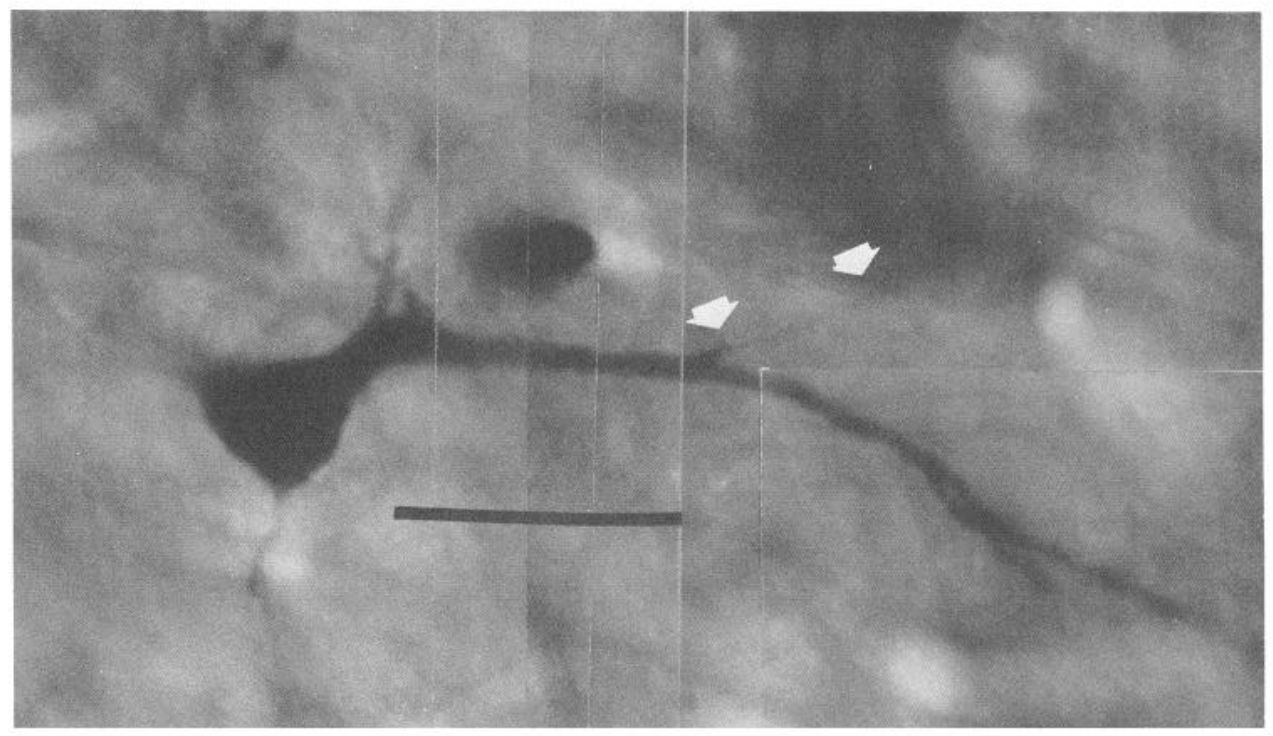

duration $(<1.5 \mathrm{msec})$, and lack the pronounced spike afterhyperpolarization (e.g., Grofová et al., 1982).

Thus, on the basis of the nature of the intracellularly recorded spike waveform and succeeding afterhyperpolarization, the pattern and rate of spontaneous activity, the occurrence of antidromic responses at long latency from neostriatum and globus pallidus, consisting usually of IS spikes only, the pars compacta neurons whose morphology is described in this report can be unambiguously identified as nigral dopaminergic projection neurons (see Figs. 10, 11).

\section{Cytoarchitecture}

The somatodendritic morphology of the HRP-filled dopaminergic neurons in our material is similar to that of mediumsized pars compacta neurons reported in previous Golgi studies of rat (Juraska et al., 1977) and primate (Schwyn and Fox, 1974) substantia nigra, or to that labeled by intracellular injection of HRP in the rat pars compacta slice (Kita et al., 1986).

Polygonal or ovoid neurons of the pars compacta were characterized by a relatively sparse dendritic tree radiating laterally,

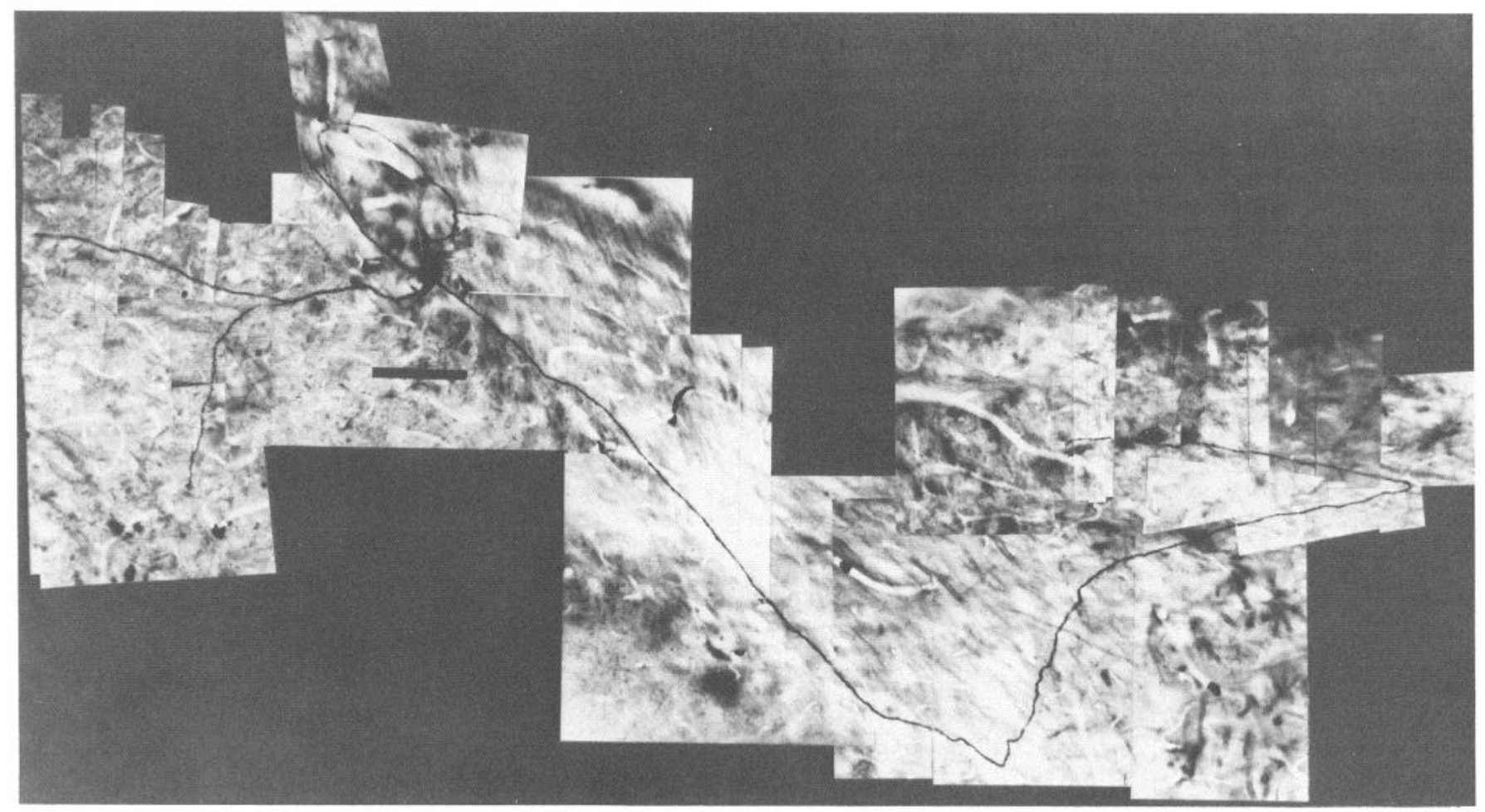

Figure 10. Photomontage constructed from different focal planes of 9 serial $60 \mu \mathrm{m}$ sections through the HRP-filled dopaminergic neuron drawn in Figure 6. Note the thick, long, and unbranched medial dendrite that follows the compacta-recticulata border until it exits the substantia nigra and continues in the adjacent ventral tegmental area. For the sake of clarity, many of the laterally directed dendrites have been truncated or excluded. Note that most of the dendrites are not beaded or varicose. Top is dorsal, left is lateral. Scale, $100 \mu \mathrm{m}$. 


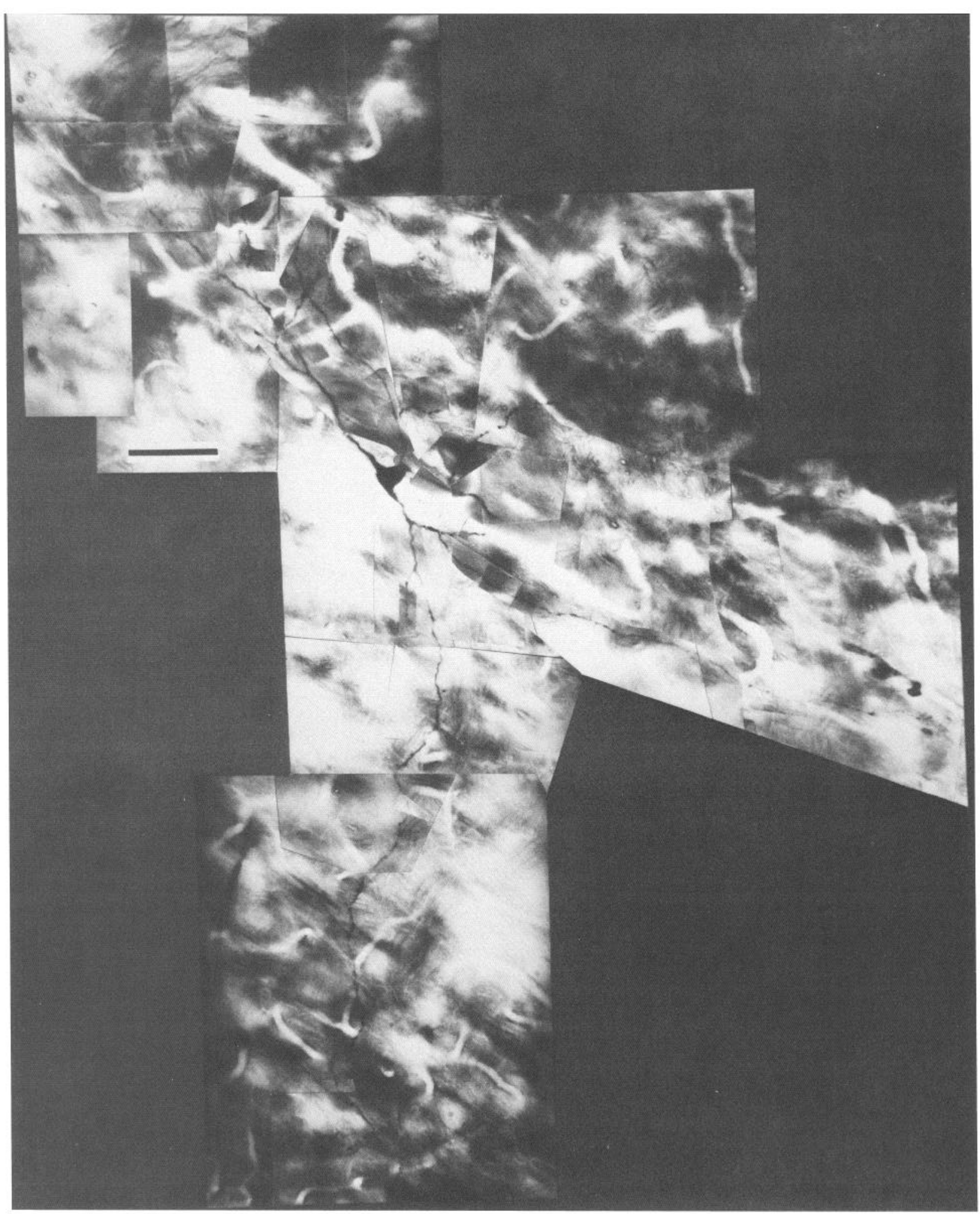

Figure 11. Photomontage constructed from different focal planes of 3 serial $60 \mu \mathrm{m}$ sections through an HRP-filled dopaminergic neuron. Top is dorsal, left is lateral. Note that most of the dendrites are not beaded or varicose. Scale, $50 \mu \mathrm{m}$.

medially, and dorsally, and located mainly within pars compacta. Each neuron also emitted 1 or 2 dendrites that traveled ventrally or ventrolaterally into pars reticulata. The thick primary dendrites usually branched within 30 or $40 \mu \mathrm{m}$ of the cell body, and gave rise to a sparse arbor of thinner secondary and tertiary dendrites.
The dorsally radiating dendrites were the shortest emitted by the neuron, rarely exceeding $250 \mu \mathrm{m}$ in length. These short dendrites have been seen previously in Golgi material (Juraska et al., 1977), but, with this technique, the possibility could not be excluded that these short dendrites derived from the incomplete nature of the reconstruction, since most Golgi studies pre- 
clude a serial reconstruction of a single neuron in its entirety. Comparison of our coronally sectioned material with the parasagittal reconstruction of an HRP-filled rat pars compacta neuron from an in vitro slice, depicted in Figure 1 of Kita et al. (1986), suggests that the short dorsal and lateral dendrites of rat compacta neurons arborize to form a relatively restricted, roughly hemispherical dendritic field, some $300-500 \mu \mathrm{m}$ in diameter, and remaining mostly within pars compacta.

The ventrally directed dendrites were usually the largest and longest emitted by compacta neurons, commonly extending for over 500 or $600 \mu \mathrm{m}$. In many cases these dendrites extended deep into pars reticulata, sometimes terminating among the fibers of the crus cerebri, as previously described for pars compacta neurons in cat (Preston et al., 1981).

Pars compacta neurons with fusiform cell bodies tended to have a slightly different dendritic orientation. In these cells, the medial and lateral dendrites predominated, although these neurons also possessed ventrally directed dendrites (cf. Fig. 5). After emerging from the medial and lateral poles of the neuron as very thick and smooth primary dendrites, these processes tapered slightly and commonly extended for $500 \mu \mathrm{m}$ or more with little branching. Dopaminergic neurons of this morphology have been previously described in rat substantia nigra as being retrogradely labeled from amygdala but not from neostriatum, and as lying dorsal to the remainder of the more typical ovoid or polygonal nigrostriatal dopaminergic cell bodies in pars compacta (Fallon et al., 1978). The same study suggested that the fusiform neurons may thus be functionally distinct from the remainder of the dopaminergic neurons. However, the fusiform neuron depicted in Figure 5 was antidromically activated from neostriatum, lending credence to the notion that the morphology of dopaminergic nigrostriatal projection neurons may be depicted as a continuum from ovoid to fusiform, with slightly different orientations of their dendritic fields, perhaps depending on their location within the nucleus, as has been shown to be the case for nondopaminergic pars reticulata neurons (Juraska et al., 1977).

In general, the dendrites of nigrostriatal and nigropallidal neurons did not cross over nuclear boundaries, a feature previously noted in Golgi studies (Juraska et al., 1977). However, in one case (cf. Figs. 6, 10), a pars compacta neuron of average size, located approximately in the middle of the medial-lateral extent of the compacta, emitted only one dendrite medially, but this process extended, unbranched, for over $1500 \mu \mathrm{m}$, closely following the ventral border of the pars compacta. This dendrite extended out of the substantia nigra proper, and well into the adjacent ventral tegmental area, where it reversed direction and turned sharply laterally before terminating in the dorsal-lateral region of the ventral tegmentum. This finding might be viewed as reinforcement for the notion that the dopaminergic neurons of the substantia nigra and ventral tegmental area are part of a single continuous cell group, rather than separate nuclei (Fallon et al., 1978; Fallon, 1981), and suggests at least the possibility of dendritic interactions between substantia nigra and ventral tegmental area neurons.

While the gencral morphology of rat substantia nigra, pars compacta neurons in the present study is comparable to that seen in other species, both the diameter and length of the dendrites of rat nigral neurons appear to be smaller than those observed in cat and primate (Schwyn and Fox, 1974; Preston et al., 1981; Phelps et al., 1983). This is unlikely to be due to a shrinkage artifact in our tissue, since all of our material was osmicated prior to dehydration in preparation for subsequent electron-microscopic analysis, in contrast to some of the former studies (e.g., Preston et al., 1981). Thus, the dopaminergic neurons of rat pars compacta are slightly smaller than corresponding neurons in cat and primate.

\section{Dendritic morphology}

In the early histofluorescence studies of dopaminergic neurons, dopaminergic dendrites appeared as highly varicose structures, and it was suggested that these varicosities might reflect the morphological substrate for the dendritic release of dopamine (Björklund and Lindvall, 1975) demonstrated by various biochemical and neuropharmacological studies (Groves et al., 1975; Geffen et al., 1976; Paden et al., 1976; Cheramy et al., 1981). Even today, most discussions of dopaminergic dendrite morphology stress the varicose nature of these dendrites. However, although some of the dendrites of the pars compacta dopaminergic neurons described in the present report had a moderately varicose appearance, the vast majority of these HRP-filled dendrites appeared nonvaricose. Moreover, during the course of the present experiments, 3 nondopaminergic pars reticulata neurons were also injected with HRP, and their dendrites were, in some cases, markedly varicose, as previously reported by others (Grofová et al., 1982). Furthermore, in previous ultrastructural studies of 5-hydroxydopamine-labeled dopaminergic dendrites, marked changes in the caliber of these dendrites, even at regions of dendrodendritic contact, were not observed even in computer-assisted 3-dimensional reconstructions of serial sections (Groves and Linder, 1983). Thus, as others have pointed out (Juraska et al., 1977; also see Fig. 3 in Schwyn and Fox, 1974), the dendritic varicosities occasionally seen in Golgi and HRP material do not correspond to the more prevalent varicosities seen in histofluorescence material, which would seem to constitute regions of relatively high accumulation of dopamine or of the histofluorescence reaction product, rather than regions of increased dendritic diameter.

While most dendrites in our material had a smooth appearance, we occasionally observed spinelike processes or dendritic blebs on dopaminergic dendrites both in pars compacta and pars reticulata, most often on the thinner, higher-order dendrites. These dendritic appendages have also been observed in Golgi material from substantia nigra in primate and cat (Schwyn and Fox, 1974; Phelps et al., 1983), but appear to be less common in rat, as evidenced by our material and that of Juraska et al. (1977).

\section{Axons and initial segments}

The axons of HRP-filled dopaminergic neurons arose from primary or proximal secondary dendrites, and appeared as very fine, smooth processes in their initial trajectory (cf. Fig. 9). The axons were so fine that precise estimates of their diameter were not attempted, since these structures appeared to be within the range of error introduced by light microscopy; rough estimates of axonal diameter would be in the range of $0.2-0.5 \mu \mathrm{m}$. No local collaterals were observed in our material, consistent with Golgi studies of rat pars compacta (Juraska ct al., 1977). It is conceivable that local collaterals are present in rat nigrostriatal neurons, but that, because of short survival times, the increased background density of our osmicated tissue, or the very fine nature of the parent axons, local axon collaterals were not wellfilled by HRP or simply escaped detection in our material.

In HRP-filled neurons of cat pars compacta, local axon col- 
laterals have been observed (Preston et al., 1981). However, these axons are considerably larger in diameter than those in the rat, despite the increased probability of shrinkage in the former study due to the lack of osmication. Consistent with this species difference in axonal caliber, antidromic conduction velocities are also much greater for cat dopaminergic neurons, being estimated at $1.4 \mathrm{~m} / \mathrm{sec}$, or almost 3 times faster than the velocity for nigrostriatal dopaminergic neurons in rat obtained in this and other studies (Deniau et al., 1978; Guyenet and Aghajanian, 1978; Grace and Bunney, 1983; Tepper et al., 1984). The absence of local collaterals in rat nigrostriatal neurons may thus be related to the species difference in axonal caliber.

\section{Functional inferences based on morphology}

Significant differences were observed between the dendritic arborization of nigral dopaminergic neurons in pars compacta and pars reticulata. Although each dopaminergic pars compacta neuron sent 1 or 2 very long dendrites into pars reticulata, a major portion of the dendritic extent of each neuron consisted of many short dendrites restricted to pars compacta and the surrounding vicinity. Anterograde labeling studies suggest that these 2 dendritic regions of the pars compacta dopamine neuron may be anatomically distinct, since the majority of neostriatal afferents to substantia nigra terminate ventrally, within pars reticulata (Hattori et al., 1975; Gerfen, 1985; Fishell and van der Kooy, 1986), whereas afferents from globus pallidus terminate preferentially in pars compacta (Hattori et al., 1975). Preliminary electophysiological data support a functional correlate to this division. Although stimulation of globus pallidus elicits IPSPs in dopaminergic nigrostriatal neurons, stimulation of the lateral dorsal neosiriatum, which elicits antidromic responses in these neurons, evokes little or no synaptic response. However, the same neostriatal stimuli readily elicit IPSPs in nondopaminergic pars reticulata neurons encountered more ventrally along the same electrode penetration (Tepper et al., 1986). It may be that the majority of basal ganglia afferents to dopaminergic neurons originate in globus pallidus and synapse along the shorter dorsal and lateral dendrites in and around pars compacta, while the longer lateral and ventral dendrites extending down into pars reticulata could serve a different function. In support of this hypothesis, preliminary ultrastructural evidence suggests that the majority of synapses onto dopaminergic neurons are located on the proximal dendrites, with many long stretches of distal dendrite extending into pars reticulata completely devoid of synaptic contact (Grofová et al., 1986). Additional information on the issue will be forthcoming from an electron-microscopic analysis of the dendrites and synaptic contacts of the neurons described in the present study. It is also possible that differences in the neurotransmitters used by the striatocompacta and striatoreticulata projections could account for the differential response to neostriatal stimulation.

Since nondopaminergic pars reticulata neurons are responsive to dopamine (Ruffieux and Schultz, 1980), and evidence exists to suggest that dopamine may modulate the effects of GABA in these cells (Waszcak and Walters, 1983), it may be the case that the long ventral dendrites of dopaminergic neurons coursing through pars reticulata function as presynaptic structures, modulating the flow of afferent information in other, nondopaminergic nigral dendrites through the release of dopamine, perhaps through dendrodendritic synapses. A similar function may obtain for the long lateral dendrites remaining in pars compacta in relation to other dopaminergic dendrites (Wilson et al., 1977a;
Groves and Linder, 1983). The significant differences in the morphology and location of individual dendrites arising from single dopaminergic neurons may reflect important differences in the function of these structures with respect to the processing of afferent input.

\section{References}

Björklund, A., and O. Lindvall (1975) Dopamine in dendrites of substantia nigra neurons. Suggestions for a role in dendritic terminals. Brain Res. 135: 315-323.

Bunney, B. S., J. R. Walters, R. H. Roth, and G. K. Aghajanian (1973) Dopaminergic neurons: Effects of antipsychotic drugs and amphetamine on single cell activity. J. Pharmacol. Exp. Ther. 185: 560-571.

Cheramy, A., V. Leviel, and J. Glowinski (1981) Dendritic release of dopamine in the substantia nigra. Nature 289: 537-542.

Deniau, J. M., C. Hammond, A. Riszk, and J. Feger (1978) Electrophysiological properties of identified output neurons of the rat substantia nigra (pars compacta and pars reticulata): Evidence for the existence of branched neurons. Exp. Brain Res. 32: 409-422.

Fallon, J. H. (1981) Collateralization of monoamine neurons: Mesotelencephalic dopamine projections to caudate septum and frontal cortex. J. Neurosci. 1: 1361-1368.

Fallon, J. H., J. N. Riley, and R. Y. Moore (1978) Substantia nigra dopamine neurons: Separate populations project to neostriatum and allocortex. Neurosci. Lett. 7: 157-162.

Fishell, G., and D. van der Kooy (1986) Developmental changes in the distribution of striatonigral terminals. Soc. Neurosci. Abstr. 12: 1544.

Geffen, L. B., T. M. Jesell, A. C. Cuello, and L. L. Iversen (1976) Release of dopamine from dendrites in rat substantia nigra. Nature 260: 258-260.

Gerfen, C. R. (1985) The neostriatal mosaic: Compartmental organization of projections from the striatum to the substantia nigra in the rat. J. Comp. Neurol. 236: 454-476.

Grace, A. A. (1987) The regulation of dopamine neuron activity as determined by in vivo and in vitro intracellular recording. In The Neurophysiology of Dopamine Systems, L. A. Chiodo and A. S. Freeman, eds., Lakeshore Press, Detroit, MI (in press).

Grace, A. A., and B. S. Bunney (1980) Nigral dopamine neurons: Intracellular recording and identification using L-dopa injection combined with fluoresence histochemistry. Science 210: 654-656.

Grace, A. A., and B. S. Bunney (1983) Intracellular and extracellular electrophysiology of nigral dopaminergic neurons. I. Identification and characterization. Neuroscience 10:301-315.

Grofová, I., J. M. Deniau, and S. T. Kitai (1982) Morphology of the substantia nigra pars reticulata projection neurons intracellularly labeled with HRP. J. Comp. Neurol. 208: 352-368.

Grofová, I., H. Kita, and S. T. Kitai (1986) Patterns of synaptic contact upon intracellularly labeled nigrostriatal neurons in the rat. Soc. Neurosci. Abstr. 12: 1542 .

Groves, P. M., and J. C. Linder (1983) Dendro-dendritic synapses in substantia nigra: Descriptions based on analysis of serial sections. Exp. Brain Res. 49: 209-217.

Groves, P. M., C. J. Wilson, S. J. Young, and G. V. Rebec (1975) Selfinhibition by dopaminergic neurons. Science 190: 522-529.

Guyenet, P. G., and G. K. Aghajanian (1978) Antidromic identification of dopaminergic and other output neurons of the rat substantia nigra. Brain Res. 150: 69-84.

Hattori, T., H. C. Fibiger, and P. L. McGeer (1975) Demonstration of a pallido-nigral projection innervating dopaminergic neurons. $J$. Comp. Neurol. 162: 487-504.

Itoh, K., A. Konishi, S. Nomura, M. Mizuno, Y. Nakamura, and T. Sugimoto (1979) Application of coupled oxidation reaction to electron microscopic demonstration of horseradish peroxidase: Cobaltglucose oxidase method. Brain Res. 175: 341-346.

Juraska, J. M., C. J. Wilson, and P. M. Groves (1977) The substantia nigra of the rat: A Golgi study, J. Comp. Neurol. 172: 565-600.

Kita, T., H. Kita, and S. T. Kitai (1986) Electrical membrane properties of rat substantia nigra compacta neurons in an in vitro slice preparation. Brain Res. 372: 21-30.

König, J. F. R., and R. A. Klippel (1963) The Rat Brain: A Stereotaxic Atlas of The Forebrain and Lower Part of the Brain Stem, Williams and Wilkins, Baltimore, MD.

Llinás, R., S. A. Greenfield, and H. Jahnsen (1984) Electrophysiology 
of pars compacta cells in the in vitro substantia nigra-a possible mechanism for dendritic release. Brain Res. 294: 127-132.

Paden, C. M., C. J. Wilson, and P. M. Groves (1976) Amphetamineinduced release of dopamine from the substantia nigra in vitro. Life Sci. 19: 1499-1506.

Phelps, P. E., A. M. Adolini, and M. S. Levine (1983) Development of the kitten substantia nigra: A rapid Golgi study of the early postnatal period. Dev. Brain Res. 10: 1-19.

Preston, R. J., R. A. McCrea, H. T. Chang, and S. T. Kitai (1981) Anatomy and physiology of substantia nigra and retrorubral neurons studied by extra- and intracellular recording and by horseradish peroxidase labeling. Neuroscience 6: 331-344.

Rinvik, E., and I. Grofová (1970) Observations on the fine structure of the substantia nigra in the cat. Exp. Brain Res. 11: 229-248.

Ruffieux, A., and W. Schultz (1980) Dopaminergic activation of reticulata neurones in the substantia nigra. Nature 285: 240-241.

Schwyn, R. C., and C. A. Fox (1974) The primate substantia nigra: A Golgi and electron microscopic study. J. Hirnforsch. 15: 95-126.

Tepper, J. M., S. Nakamura, S. J. Young, and P. M. Groves (1984)
Autoreceptor-mediated changes in dopaminergic terminal excitability: Effects of striatal drug infusions. Brain Res. 309: 317-333.

Tepper, J. M., S. F. Sawyer, S. J. Young, and P. M. Groves (1986) Intracellular recording and HRP staining of rat nigral neurons. Soc. Neurosci. Abstr. 12: 1542.

Waszcak, B. L., and J. R. Walters (1983) Dopamine modulation of the effects of gamma-amino butyric acid on substantia nigra pars reticulata neurons. Science 220: 218-221.

Wilson, C. J., and P. M. Groves (1979) A simple and rapid section embedding technique for sequential light and electron microscopic examination of individually stained central neurons. J. Neurosci. Methods 1: 383-391.

Wilson, C. J., P. M. Groves, and E. Fifková (1977a) Monoaminergic synapses, including dendro-dendritic synapses in the rat substantia nigra. Exp. Brain Res. 30: 161-174.

Wilson, C. J., S. J. Young, and P. M. Groves (1977b) Statistical properties of neuronal spike trains in the substantia nigra: Cell types and their interactions. Brain Res. 36: 243-260. 